\section{Avaliação do conhecimento sobre níveis tensionais e cronicidade da hipertensão: estudo com usuários de uma Farmácia Básica no Sul do Brasil}

\author{
Evaluation of knowledge on blood pressure levels \\ and chronicity of hypertension among users of a \\ public pharmacy in Southern Brazil
}

\author{
Evaluación de los conocimientos acerca de \\ los niveles de presión y de la cronicidad de la \\ hipertensión arterial: estudio con usuarios de una \\ Farmacia Comunitaria en el Sul de Brasil
}

\begin{abstract}
The aim of this study was to evaluate patients' knowledge on hypertension and associated factors through a cross-sectional study of adults using antihypertensive medication dispensed by the São Francisco de Paula Public Pharmacy in Rio Grande do Sul State, Brazil. The study evaluated knowledge on the normal limits for systolic and diastolic blood pressure and chronicity of hypertension. Multinomial regression was used to measure knowledge (satisfactory/moderatel unsatisfactory); satisfactory was defined as correct knowledge on two or more items. Of the 635 users with hypertension, $27.7 \%$ showed satisfactory knowledge, while $47.2 \%$ were only aware of the chronic nature of hypertension. After adjustment, female gender, more education, not living alone, healthy lifestyle, and greater time since diagnosis were associated with satisfactory knowledge on hypertension. Considering the importance of patient autonomy in monitoring blood pressure and continuous treatment to control hypertension, the study showed a low prevalence of satisfactory knowledge.
\end{abstract}

Antihypertensive Agents; Primary Health Care; Arterial Pressure; Health Knowledge, Attitudes, Practice
Fabiane Raquel Motter 1

Maria Teresa Anselmo Olinto 1,2 Vera Maria Vieira Paniz 1

\section{Resumo}

Objetivou-se avaliar o conhecimento sobre hipertensão arterial sistêmica (HAS) e fatores associados, por meio de estudo transversal com adultos usuários de medicamentos anti-hipertensivos adquiridos na Farmácia Básica de São Francisco de Paula, Rio Grande do Sul, Brasil. Avaliou-se o conhecimento sobre os limites de normalidade para a pressão arterial sistólica e diastólica e sobre a condição crônica da HAS. Utilizou-se regressão multinomial para conhecimento (satisfatório/moderado/insatisfatório) $e$ considerou-se como satisfatório o conhecimento sobre dois ou mais aspectos avaliados. Dos 635 usuários, portadores de HAS, 27,7\% apresentaram conhecimento satisfatório e 47,2\% conheciam apenas a cronicidade da HAS. Após ajuste, sexo feminino, maior escolaridade, não morar só, comportamento saudável e maior tempo de diagnóstico associaram-se ao conhecimento satisfatório sobre a HAS. Considerando a importância da autonomia do portador no monitoramento dos níveis tensionais e da continuidade do tratamento no controle da pressão arterial, encontrou-se baixa prevalência de conhecimento satisfatório.

Antihipertensivos; Atenção Primária à Saúde; Pressão Arterial; Conhecimento, Atitudes e Práticas em Saúde 


\section{Introdução}

A hipertensão arterial sistêmica (HAS) é um importante problema de saúde pública, uma vez que apresenta elevada prevalência e está associada a $40 \%$ das mortes por acidente vascular cerebral, $25 \%$ das mortes por doença coronariana aguda e, em combinação com o diabetes, por $50 \%$ dos casos de insuficiência renal $1,2,3$. No Brasil, estudos de base populacional estimam que essa morbidade afete entre $20 \%$ e $30 \%$ da população adulta 4,5,6.

Diante desse contexto, observa-se o controle dos níveis tensionais (valores inferiores a 140mmHg de pressão arterial sistólica - PAS e a 90mmHg de pressão arterial diastólica - PAD) como importante condição para a redução da morbidade e mortalidade cardiovascular. Dados apontam uma relação contínua entre níveis tensionais e taxas de mortalidade cardiovascular, demonstrando ainda que uma redução média de $5 \mathrm{mmHg}$ na pressão arterial diastólica (e/ou $10 \mathrm{mmHg}$ na pressão sistólica) diminui o risco de acidente vascular cerebral em aproximadamente um terço e o risco de doença arterial coronariana em um sexto ${ }^{\text {? }}$.

O tratamento da HAS abrange duas abordagens terapêuticas: o tratamento não farmacológico baseado em modificações do estilo de vida (perda de peso, incentivo às atividades físicas, alimentação saudável, entre outros) e o tratamento farmacológico 8 . Entretanto, pesquisas revelam que somente metade dos portadores de HAS possui níveis tensionais controlados. Existem evidências de que esses tendem a interromper o tratamento quando apresentam níveis tensionais controlados, por não associarem o efeito do tratamento contínuo à manutenção dos níveis pressóricos. Por outro lado, portadores que aderem às medidas terapêuticas podem apresentar redução insuficiente dos níveis tensionais e não perceberem devido à ausência de sintomas.

Estudos mostram que portadores de HAS que possuem conhecimento sobre a sua morbidade apresentam maior autonomia no monitoramento dos níveis pressóricos 9 , aderem melhor ao tratamento e, consequentemente, possuem níveis tensionais mais controlados 10,11,12. Nesse sentido, objetivou-se avaliar o conhecimento de portadores sobre HAS abordando os limites de normalidade para pressão sistólica e diastólica e a condição crônica desta morbidade, identificando ainda, os fatores associados ao conhecimento.

\section{Metodologia}

Realizou-se um estudo transversal com adultos de 20 anos ou mais de idade, usuários da Farmácia Básica do Município de São Francisco de Paula, Rio Grande do Sul, Brasil. Essa farmácia pertence ao serviço público de saúde, sendo a única que fornece medicamentos gratuitamente no município. Foram incluídos no estudo os usuários com pelo menos um dos seguintes medicamentos prescritos e dispensados: captopril $25 \mathrm{mg}$, espironolactona $25 \mathrm{mg}$, furosemida $40 \mathrm{mg}$, hidroclorotiazida $25 \mathrm{mg}$, metildopa $250 \mathrm{mg}$, tartarato de metoprolol 100mg, nifedipina 10 e 20mg, propranolol 40mg e verapamil $80 \mathrm{mg}$. Foram excluídos os indivíduos com déficit cognitivo ou em terapia de substituição renal. Considerando o objetivo de avaliar o conhecimento sobre a HAS, incluíram-se nesta análise apenas os indivíduos que referiram ter o diagnóstico médico de HAS por meio da pergunta "Algum médico já disse que o(a) senhor (a) tem pressão alta ou hipertensão arterial sistêmica?".

Realizou-se um processo de amostragem de forma consecutiva e não aleatória de usuários que acessaram a farmácia no período de novembro de 2010 a fevereiro de 2011 . Aqueles que preenchiam os critérios de inclusão eram, imediatamente após a dispensação do medicamento, convidados a participar da pesquisa. No caso de recusa, a pesquisadora realizava novamente o convite a cada dispensação ocorrida no período do estudo. O usuário era entrevistado uma única vez, ainda que voltasse a utilizar o serviço da farmácia básica no período da investigação. Quatro entrevistadoras treinadas realizaram as entrevistas por meio da aplicação de questionário padronizado, pré-codificado e pré-testado em estudo piloto. Todos os questionários foram revisados e codificados logo após as entrevistas. O detalhamento sobre a metodologia está descrito em outra publicação ${ }^{13}$.

A amostra incluiu 635 usuários hipertensos e permitiu estimar uma prevalência de conhecimento sobre a HAS de $50 \%$, com margem de erro de $\pm 3,5$ pontos percentuais. Permitiu, ainda, detectar razões de prevalência de 1,8 ou maiores, com poder estatístico de $80 \%$, utilizando-se um nível de 95\% de confiança.

As variáveis sociodemográficas avaliadas foram sexo, idade, cor da pele (observada e classificada em branca ou não branca pela entrevistadora), escolaridade e situação de moradia (morar só ou não). Como variáveis comportamentais investigaram-se o hábito de fumar (fumante, exfumante e nunca fumou) e o consumo de bebidas alcoólicas (não consome, consumo menor ou igual a $15 \mathrm{~g}$ de álcool/dia e consumo maior 
que $15 \mathrm{~g} /$ dia) 8 . Investigou-se, também, a prática de atividade física no lazer 8 (prática de alguma atividade física semanalmente por pelo menos 30 minutos por dia, três vezes/semana) e o consumo diário de frutas e verduras 8,14,15, os quais foram utilizados para a construção da variável comportamento saudável (indivíduo que pratica no mínimo 30 minutos de alguma atividade física por pelo menos três vezes por semana e que consome diariamente frutas e verduras) com o objetivo de mensurar modificações de estilo de vida recomendadas para a redução da pressão arterial ${ }^{8}$. Como variáveis de saúde, avaliou-se a autopercepção de saúde (excelente, muito boa, boa, regular e ruim, categorizada em boa/muito boa/excelente e ruim/regular), comorbidades (categorizadas em nenhuma, cardiovasculares e outras) e tempo em anos de diagnóstico de HAS. Investigaram-se, ainda, as variáveis: número de consultas médicas e consultar o mesmo médico para HAS.

Para avaliar o conhecimento sobre a HAS abordou-se os seguintes aspectos: limites de normalidade para a PAS e PAD e sobre a condição crônica da HAS. O conhecimento sobre níveis tensionais foi estabelecido por meio da pergunta previamente testada em estudo piloto: “A partir de que valor de pressão o(a) senhor(a) considera uma pessoa com pressão alta ou hipertensa?". Utilizou-se como valores de referência os níveis tensionais preconizados pelas VIII Diretrizes Brasileiras de Hipertensão, sendo igual a $140 \mathrm{mmHg}$ para pressão sistólica e $90 \mathrm{mmHg}$ para pressão diastólica 8.

Para a investigação do conhecimento sobre a condição crônica da HAS foi feita a seguinte questão: "A pressão alta ou hipertensão é uma doença que dura?", e as alternativas de respostas eram lidas para o entrevistado. Considerouse como correta a alternativa que descrevia que a HAS é uma doença que dura por toda vida. O desfecho foi categorizado em indivíduos com conhecimento satisfatório (conhecem dois ou mais aspectos avaliados), moderado (conhecem um dos aspectos avaliados) e insatisfatório (não conhecem nenhum dos aspectos avaliados) para a autonomia no monitoramento dos níveis pressóricos e para a manutenção do tratamento contínuo.

A digitação dos dados foi realizada no programa EpiData 3.0 (EpiData Association, Odense, Dinamarca) com checagem automática de consistência e dupla digitação para correção dos possíveis erros. A análise dos dados foi feita no programa estatístico Stata 9.0 (Stata Corp., College Station, Estados Unidos).

A análise descritiva caracterizou os usuários conforme as variáveis sociodemográficas, comportamentais, de saúde e de utilização de serviços de saúde. As prevalências de conhecimento que constituíram o desfecho foram também descritas e analisadas individualmente por meio do teste do qui-quadrado. Para as análises bruta e ajustada, utilizou-se a regressão multinomial, considerando os indivíduos com conhecimento insatisfatório como categoria de referência. Embora o desfecho em estudo tenha uma aparente ordenação, sugestiva de análise pelo método de regressão logística ordinal, sua utilização pressupõe uma variável resposta original contínua que foi posteriormente agrupada e uma suposição de chances proporcionais válida, mediante teste de Brant 16,17. No presente estudo, o modelo ordinal não apresentou paralelismo para algumas covariáveis, indicando violação da premissa principal. Além disso, a variável resposta teve origem em uma variável discreta. Assim, utilizou-se regressão multinomial por ser um método estatístico adequado para desfechos com mais de duas categorias, em que cada uma é comparada à de referência em um único processo. Desse modo, informa a probabilidade de cada indivíduo estar em uma determinada categoria de acordo com a variável independente por meio do comando $r r$, que estima razões de prevalência em modelos multivariados no programa estatístico Stata. Todas as variáveis que apresentaram associação $\mathrm{p} \leq 0,2$ na análise bruta foram incluídas na análise multivariada. Com o objetivo de controlar para possíveis fatores de confusão, um modelo hierarquizado com quatro níveis foi elaborado e as variáveis foram controladas para aquelas do mesmo nível ou superior, sendo mantidas no modelo aquelas com valor de $\mathrm{p} \leq 0,218$. No nível mais distal encontram-se as variáveis sociodemográficas: sexo, idade, escolaridade e situação de moradia. No segundo nível foram incluídas as variáveis comportamentais: hábito de fumar e comportamento saudável. No terceiro, encontram-se as variáveis comorbidades e tempo de HAS. As variáveis número de consultas médicas e consultar com o mesmo médico foram avaliadas como determinantes proximais do conhecimento sobre a HAS.

Esta pesquisa foi aprovada pelo Comitê de Ética em Pesquisa da Universidade do Vale dos Sinos (UNISINOS; CEP 10/133). Os participantes assinaram um Termo de Consentimento para a realização da entrevista. Foi garantido o sigilo quanto à identidade e confidencialidade das informações, bem como o direito de não participar ou suspender a participação a qualquer momento. 


\section{Resultados}

Foram entrevistados 678 usuários de medicamentos para o tratamento da HAS, dispensados na Farmácia Básica do Município de São Francisco de Paula. Desses, 635 (93,7\%) referiram ser portadores de HAS e foram incluídos na análise deste estudo.

Os usuários, portadores de HAS, incluídos neste trabalho apresentaram, em média, 58 anos de idade ( $\mathrm{DP}=12,2)$, eram predominantemente do sexo feminino $(67,7 \%)$ e de cor da pele branca $(72,3 \%)$. Observou-se que metade possuía até quatro anos de estudos e $85 \%$ não moravam sozinhos. Quanto às variáveis comportamentais, verificou-se que cerca de $50 \%$ dos portadores consumiam diariamente frutas e verduras, e menos de um terço praticava atividade física regularmente.

Em relação à presença de comorbidades, 1/5 referiu doenças cardiovasculares. Verificou-se, ainda, que $44,8 \%$ dos portadores foram diagnosticados com HAS no período inferior a cinco anos e que mais da metade costumava consultar o mesmo médico para o tratamento da HAS.

A Tabela 1 descreve a prevalência de conhecimento sobre os limites de normalidade para PAS e PAD e a cronicidade da HAS. Observa-se que somente $27,7 \%$ dos portadores mostraram conhecimento satisfatório, a maioria $(54,7 \%)$ conhece somente um dos aspectos avaliados, e

Tabela 1

Prevalência de conhecimento (satisfatório/moderado/ insatisfatório) sobre hipertensão arterial sistêmica (HAS) em usuários hipertensos da Farmácia Básica do Município de São Francisco de Paula, Rio Grande do Sul, Brasil, 2011 $(\mathrm{N}=635)$.

\begin{tabular}{lcc}
\hline Conhecimento & $\mathbf{n}$ & $\%$ \\
\hline Satisfatório & 176 & 27,7 \\
Cronicidade, PAS e PAD & 21 & 3,3 \\
Cronicidade e PAS & 117 & 18,4 \\
Cronicidade e PAD & 32 & 5,0 \\
PAS e PAD & 6 & 0,9 \\
Moderado & 347 & 54,7 \\
Cronicidade & 300 & 47,3 \\
PAS & 39 & 6,1 \\
PAD & 8 & 1,3 \\
Insatisfatório & 112 & 17,6 \\
\hline
\end{tabular}

PAD: pressão arterial diastólica; PAS: pressão arterial sistólica. somente 3,3\% conhecem todos. Entre os três aspectos avaliados a maior proporção do conhecimento é representada pela cronicidade da HAS.

A Tabela 2 descreve a distribuição dos aspectos do conhecimento sobre HAS conforme as variáveis sociodemográficas, comportamentais, de saúde e de utilização de serviços de saúde. Não houve diferença estatisticamente significativa entre as categorias do desfecho e as variáveis cor da pele, hábito de fumar, consumo de álcool e número de consultas médicas. Maiores prevalências de conhecimento satisfatório sobre a HAS foram observadas em mulheres, entre $54 \mathrm{e}$ 65 anos, com pelo menos o primeiro grau completo, que praticam atividade física regularmente e consomem diariamente frutas e/ou verduras.

A Tabela 3 mostra os resultados obtidos com base na análise multinominal ajustada utilizando o conhecimento insatisfatório como categoria de referência. Observou-se que mulheres, indivíduos que não moram sozinhos e que relataram comportamento saudável associaram-se a um conhecimento satisfatório sobre HAS. As variáveis escolaridade e tempo de diagnóstico de HAS apresentaram associação linear direta tanto com o conhecimento moderado quanto com o satisfatório. Indivíduos com maior tempo de diagnóstico tiveram uma probabilidade 4,6 vezes maior de apresentar um conhecimento satisfatório em relação àqueles com cinco anos ou menos de diagnóstico de HAS. O número de consultas médicas não esteve associado a um maior conhecimento, mesmo após o controle para o tempo de doença, e consultar com o mesmo médico apresentou uma probabilidade $80 \%$ maior de conhecimento, sendo esta associação limítrofe para o conhecimento satisfatório.

\section{Discussão}

O presente trabalho verificou baixa prevalência de conhecimento satisfatório para a autonomia do portador de HAS no monitoramento dos níveis pressóricos e para a manutenção do tratamento contínuo como forma de controle da pressão arterial. Sexo feminino, maior escolaridade, não morar sozinho, possuir um comportamento saudável e maior tempo de diagnóstico estão associados ao conhecimento satisfatório sobre a HAS.

Quanto aos aspectos que constituíram o desfecho, percebe-se que o conhecimento sobre a cronicidade foi superior em relação aos limites de normalidade de pressão arterial. Cerca de três quartos dos entrevistados relataram que a HAS é uma doença para toda vida. Esse resultado é semelhante aos encontrados nas literaturas 
Tabela 2

Prevalência de conhecimento (satisfatório/moderado/insatisfatório) sobre hipertensão arterial sistêmica (HAS) conforme as variáveis sociodemográficas, comportamentais, de saúde e de utilização de serviços de saúde em usuários hipertensos da Farmácia Básica do Município de São Francisco de Paula, Rio Grande do Sul, Brasil, 2011.

\begin{tabular}{|c|c|c|c|c|c|}
\hline Variável & $\mathbf{n}$ & $\begin{array}{c}\text { Conhecimento } \\
\text { insatisfatório } \\
n(\%)\end{array}$ & $\begin{array}{l}\text { Conhecimento } \\
\text { moderado } \\
\text { n (\%) }\end{array}$ & $\begin{array}{c}\text { Conhecimento } \\
\text { satisfatório } \\
\text { n (\%) }\end{array}$ & $\begin{array}{l}\text { Valor } \\
\text { de } p \text { * }\end{array}$ \\
\hline Total & 635 & $112(17,7)$ & $347(54,7)$ & $176(27,7)$ & \\
\hline Sexo & & & & & 0,022 \\
\hline Masculino & 205 & $46(22,4)$ & $114(55,6)$ & $45(21,2)$ & \\
\hline Feminino & 430 & $66(15,4)$ & $233(54,2)$ & $131(30,5)$ & \\
\hline Idade (anos) & & & & & 0,037 \\
\hline$>65$ & 181 & $35(15,0)$ & $131(56,0)$ & $68(29,0)$ & \\
\hline $54-65$ & 220 & $33(15,0)$ & $118(53,4)$ & $69(31,4)$ & \\
\hline$\leq 53$ & 234 & $44(24,3)$ & $98(54,2)$ & $39(21,6)$ & \\
\hline Cor da pele & & & & & 0,570 \\
\hline Branca & 459 & $77(16,8)$ & $256(55,8)$ & $126(27,5)$ & \\
\hline Não branca & 176 & $35(19,9)$ & $91(51,7)$ & $50(28,4)$ & \\
\hline Escolaridade (anos de estudos) & & & & & $<0,001$ \\
\hline 0 & 91 & $28(30,7)$ & $50(55,0)$ & $13(14,3)$ & \\
\hline $1-4$ & 219 & $42(19,2)$ & $122(55,7)$ & $55(25,1)$ & \\
\hline $5-8$ & 238 & $33(13,9)$ & $135(56,7)$ & $70(29,4)$ & \\
\hline 9 ou mais & 87 & $9(10,4)$ & $40(46,0)$ & $38(43,7)$ & \\
\hline Situação de moradia & & & & & 0,048 \\
\hline Mora só & 91 & $23(25,3)$ & $50(55,0)$ & $18(19,8)$ & \\
\hline Não mora só & 541 & $88(16,3)$ & $295(54,5)$ & $158(29,2)$ & \\
\hline Hábito de fumar & & & & & 0,087 \\
\hline Não fumante & 297 & $63(21,2)$ & $148(49,8)$ & $86(29,0)$ & \\
\hline Ex-fumante & 206 & $32(15,5)$ & $116(56,3)$ & $58(28,2)$ & \\
\hline Fumante & 132 & $17(12,9)$ & $83(62,9)$ & $32(24,2)$ & \\
\hline Consumo de álcool & & & & & 0,155 \\
\hline Não consome & 515 & $96(18,6)$ & $279(54,2)$ & $140(27,2)$ & \\
\hline Consome $\leq 15 \mathrm{~g} / \mathrm{dia}$ & 82 & $8(9,7)$ & $45(54,8)$ & $29(35,4)$ & \\
\hline Consome $>15 \mathrm{~g} / \mathrm{dia}$ & 38 & $8(21,5)$ & $23(60,5)$ & $7(18,4)$ & \\
\hline Atividade física & & & & & 0,011 \\
\hline Pratica & 181 & $26(14,4)$ & $90(49,7)$ & $65(35,9)$ & \\
\hline Não pratica & 452 & $86(19,0)$ & $256(56,7)$ & $110(24,3)$ & \\
\hline Consumo de frutas & & & & & 0,024 \\
\hline Sempre & 300 & $65(15,1)$ & $238(55,1)$ & $129(29,9)$ & \\
\hline$\leq 6$ vezes/semana & 330 & $47(23,2)$ & $109(53,7)$ & $47(23,2)$ & \\
\hline Consumo de verduras & & & & & 0,009 \\
\hline Sempre & 432 & $41(13,7)$ & $161(53,7)$ & $98(32,7)$ & \\
\hline$\leq 6$ vezes/semana & 203 & $69(20,9)$ & $183(55,5)$ & $78(23,6)$ & \\
\hline Comportamento saudável ** & & & & & $<0,001$ \\
\hline $\operatorname{Sim}$ & 101 & $9(9,0)$ & $46(45,5)$ & $46(45,5)$ & \\
\hline Não & 527 & $101(19,2)$ & $297(56,4)$ & $129(24,5)$ & \\
\hline
\end{tabular}

(continua) 
Tabela 2 (continuação)

\begin{tabular}{|c|c|c|c|c|c|}
\hline Variável & $\mathbf{n}$ & $\begin{array}{c}\text { Conhecimento } \\
\text { insatisfatório } \\
\text { n (\%) }\end{array}$ & $\begin{array}{c}\text { Conhecimento } \\
\text { moderado } \\
\text { n (\%) }\end{array}$ & $\begin{array}{c}\text { Conhecimento } \\
\text { satisfatório } \\
\text { n (\%) }\end{array}$ & $\begin{array}{l}\text { Valor } \\
\text { de } p \text { * }\end{array}$ \\
\hline Autopercepção da saúde & & & & & 0,671 \\
\hline Boa/Muito boa/Excelente & 221 & $35(15,8)$ & $124(56,1)$ & $62(28,1)$ & \\
\hline Regular/Ruim & 413 & $77(18,7)$ & $222(53,8)$ & $114(27,6)$ & \\
\hline Comorbidades & & & & & 0,027 \\
\hline Nenhuma & 107 & $18(16,8)$ & $65(60,8)$ & $24(22,4)$ & \\
\hline Cardiovasculares & 123 & $31(25,2)$ & $66(53,7)$ & $26(21,1)$ & \\
\hline Outras & 402 & $61(15,2)$ & $216(53,7)$ & $125(31,1)$ & \\
\hline Tempo de diagnóstico de & & & & & $<0,001$ \\
\hline \multicolumn{6}{|l|}{ hipertensão (anos) } \\
\hline$\leq 5$ & 270 & $64(23,7)$ & $143(53,0)$ & $63(23,3)$ & \\
\hline $6-10$ & 169 & $28(16,6)$ & $89(52,7)$ & $52(30,8)$ & \\
\hline$\geq 11$ & 164 & $12(7,3)$ & $99(60,4)$ & $53(32,3)$ & \\
\hline 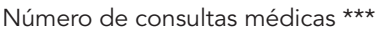 & & & & & 0,523 \\
\hline $0-1$ & 45 & $4(8,9)$ & $31(68,9)$ & $31(25,8)$ & \\
\hline $2-3$ & 208 & $33(15,9)$ & $111(53,4)$ & $61(29,5)$ & \\
\hline $4-6$ & 207 & $36(17,4)$ & $110(53,1)$ & $64(30,8)$ & \\
\hline 7 ou mais & 120 & $22(18,3)$ & $67(55,8)$ & $10(22,2)$ & \\
\hline Consultar o mesmo médico & & & & & 0,025 \\
\hline Sim & 370 & $52(14,1)$ & $206(55,7)$ & $112(30,3)$ & \\
\hline Não & 247 & $55(22,3)$ & $130(52,3)$ & $62(25,1)$ & \\
\hline
\end{tabular}

* Valor de $p$ do teste qui-quadrado para heterogeneidade de proporções;

** Indivíduo que pratica no mínimo 30 minutos de alguma atividade física por pelo menos três vezes por semana e que

consome diariamente frutas e verduras;

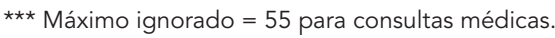

nacional 19 e internacional 20,21,22,23. Em relação ao conhecimento sobre os valores limítrofes dos níveis tensionais, verificou-se baixa prevalência de conhecimento, uma vez que $28,7 \%$ e $10,5 \%$ conheciam os limites de normalidade para PAS e $\mathrm{PAD}$, respectivamente. Estudos revelam resultados superiores em relação a esse conhecimento $19,20,21,22,24,25,26,27,28$. As diferenças encontradas podem estar relacionadas às distintas metodologias utilizadas para a avaliação do conhecimento. Dados mostram que o conhecimento sobre os limites de normalidade de pressão encoraja o monitoramento dos valores pressóricos, promovendo, assim, uma significativa redução destes 9 .

Analisando os fatores associados ao conhecimento, constatou-se uma maior prevalência de conhecimento no sexo feminino. Esse achado é consistente com o de outros estudos, que demonstram que as mulheres têm maior preocupação com sua saúde, apresentando maior tendência a utilizar os serviços de saúde e ao autocuidado 29,30.
A escolaridade esteve diretamente associada a um maior conhecimento sobre a HAS. Essa tendência está bem estabelecida pela literatura $22,23,28,31,32$, uma vez que a escolaridade influencia no grau de compreensão das informações e favorece a interação do usuário com o profissional de saúde durante a consulta médica, no que diz respeito às suas necessidades em saúde.

A análise também mostrou uma maior prevalência de conhecimento sobre a HAS em portadores que relataram não morar sozinhos. Não foram encontrados outros estudos investigando tal associação. Entretanto, pesquisa realizada por Morgado et al. 12 mostrou que portadores que moram sozinhos possuem cinco vezes mais chances de apresentar um controle inadequado da HAS. Uma explicação para esse achado é a participação da família no controle de morbidades crônicas, visto que constitui uma rede de apoio para o portador em relação aos cuidados de saúde. 
Tabela 3

Análise multinomial ajustada do conhecimento (satisfatório/moderado/insatisfatório) sobre hipertensão arterial sistêmica (HAS) em usuários hipertensos da Farmácia Básica do Município de São Francisco de Paula, Rio Grande do Sul, Brasil, 2011 $(\mathrm{N}=635)$.

\begin{tabular}{|c|c|c|}
\hline \multirow[t]{2}{*}{ Variável * } & $\begin{array}{l}\text { Conhecimento } \\
\text { moderado }\end{array}$ & Conhecimento satisfatório \\
\hline & RP (IC95\%) & RP (IC95\%) \\
\hline \multicolumn{3}{|l|}{ Nível 1} \\
\hline Sexo & $\begin{array}{l}p=0,094 * * \\
1,00\end{array}$ & $\begin{array}{l}p=0,009 * * \\
1,00\end{array}$ \\
\hline Masculino & $1,47(0,94-2,29)$ & $2,01(1,19-3,39)$ \\
\hline $\begin{array}{l}\text { Feminino } \\
\text { Escolaridade (anos de estudos) }\end{array}$ & $p=0,010 * \star \star$ & 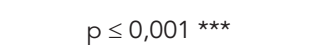 \\
\hline $\begin{array}{l}\text { Escolaridade (anos de estudos) } \\
\qquad\end{array}$ & 1,00 & 1,00 \\
\hline $1-4$ & $1,61(0,89-2,91)$ & $2,85(1,30-6,24)$ \\
\hline $5-8$ & $2,22(1,21-4,08)$ & $4,55(2,06-10,02)$ \\
\hline 9 ou mais & $2,35(0,99-5,58)$ & $8,45(3,14-22,76)$ \\
\hline Situação de moradia & $\begin{array}{c}p=0,209 * * \\
1,00\end{array}$ & $\begin{array}{c}p=0,049 * * \\
1,00\end{array}$ \\
\hline $\begin{array}{l}\text { Mora só } \\
\text { Não mora só }\end{array}$ & $1,43(0,82-2,49)$ & $2,00(1,01-3,98)$ \\
\hline \multicolumn{3}{|l|}{ Nível 2} \\
\hline Hábito de fumar & $p=0,004 * \star$ & $p=0,099 * *$ \\
\hline Não fumante & 1,00 & 1,00 \\
\hline Ex-fumante & $1,83(1,08-3,11)$ & $1,63(0,90-2,96)$ \\
\hline Fumante & $2,21(1,20-4,10)$ & $1,62(0,80-3,26)$ \\
\hline Comportamento saudável \# & $p=0,230 * *$ & $p=0,005 * *$ \\
\hline Sim & $1,61(0,74-3,50)$ & $3,16(1,42-7,01)$ \\
\hline Não & 1,00 & 1,00 \\
\hline \multicolumn{3}{|l|}{ Nível 3} \\
\hline Comorbidades & 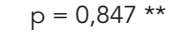 & $p=0,077 \star *$ \\
\hline Nenhuma & 1,00 & 1,00 \\
\hline Cardiovasculares & $0,54(0,26-1,13)$ & $0,48(0,19-1,17)$ \\
\hline Outras & $0,90(0,47-1,73)$ & $1,42(0,67-3,01)$ \\
\hline Tempo de diagnóstico de hipertensão (anos) & $p \leq 0,001 * * \star$ & $p \leq 0,001 \star \star \star$ \\
\hline$\leq 5$ & 1,00 & 1,00 \\
\hline $6-10$ & $1,49(0,86-2,60)$ & $1,90(1,02-3,57)$ \\
\hline$\geq 11$ & $4,30(2,08-8,89)$ & $4,66(2,12-10,25)$ \\
\hline \multicolumn{3}{|l|}{ Nível 4} \\
\hline Consultar o mesmo médico & $p=0,023 \star \star$ & $p=0,057 * \star$ \\
\hline $\operatorname{Sim}$ & $1,79(1,08-2,95)$ & $1,73(0,98-3,05)$ \\
\hline Não & 1,00 & 1,00 \\
\hline
\end{tabular}

IC95\%: intervalo de 95\% de confiança; RP: razão de prevalência.

* Cada variável está ajustada para as demais do mesmo nível e para as dos níveis acima (foram mantidas no modelo as variáveis com valor de $\mathrm{p} \leq 0,2$ );

** Teste de Wald para heterogeneidade;

*** Teste de Wald para tendência linear;

\# Indivíduo que pratica no mínimo 30 minutos de alguma atividade física por pelo menos três vezes por semana e que consome diariamente frutas e verduras. 
Outro fator associado ao conhecimento foi o comportamento saudável, mostrando que portadores que praticam atividade física e consomem frutas e verduras diariamente possuem maior prevalência de conhecimento satisfatório sobre a HAS. Embora a literatura não apresente dados sobre essa associação, um estudo realizado por Strummer et al. 33 mostrou que portadores de HAS que não possuem hábitos saudáveis apresentam elevadas prevalências de manejo inadequado desta morbidade. Nessa direção, Prugger et al. ${ }^{34}$ verificaram que portadores de HAS que recebem orientação médica sobre mudanças no estilo de vida possuem um melhor conhecimento sobre valores limítrofes da pressão arterial. Em adição, esse achado é coerente com outro trabalho que verificou um efeito de cluster para comportamento saudável, revelando que indivíduos que aderem ao padrão alimentar vegetais/frutas e atividade física de lazer têm uma atitude positiva sobre a sua saúde 35 .

No presente estudo, observou-se, também, uma associação direta entre o tempo de diagnóstico e o conhecimento sobre a HAS. Esse resultado é consistente com a literatura, uma vez que pesquisas mostram que quanto maior o tempo decorrido do diagnóstico de HAS, maiores as prevalências de conhecimento sobre esta morbidade 22,36. O maior tempo de diagnóstico pressupõe maior utilização dos serviços de saúde, proporcionando também maior exposição do portador às orientações necessárias para o controle da HAS. Entretanto, mesmo após ajuste para o tempo de diagnóstico, o número de consultas médicas não apresentou associação com o conhecimento sobre HAS, sugerindo falhas na abordagem das orientações médicas para a completa compreensão do usuário, independentemente da sua escolaridade. Apesar disso, verificou-se associação limítrofe entre consultar o mesmo médico e maior conhecimento sobre a HAS. Esse achado sugere que a existência de uma relação duradoura entre médico e paciente pode contribuir para a qualidade das orientações prestadas durante a consulta médica, como também a compreensão das informações recebidas.

Considerando-se algumas limitações metodológicas presentes, destaca-se que estudos com delineamento transversal estão sujeitos à causalidade reversa, pois exposição e desfecho são medidos no mesmo momento. Neste trabalho, esse efeito pode ter afetado a associação da variável consultar o mesmo médico com o conhecimento, uma vez que o vínculo entre médico e paciente pode melhorar o conhecimento, mas, por outro lado, a consulta com um médico regular poderia ser consequência deste. Outro aspecto a destacar se refere à generalização dos achados. A amostra estudada é composta por usuários da atenção básica que obtém seus medicamentos de forma gratuita, permitindo a generalização dos resultados para esta população. Outro aspecto a destacar diz respeito à análise por regressão multinomial estimada por razões de prevalência, que pode conduzir a uma superestimação da medida de efeito em comparação a outros modelos alternativos de análise propostos recentemente 37 .

A classificação do conhecimento aqui proposta considerou dois aspectos fundamentais no manejo da HAS: a autonomia do paciente no monitoramento de seus níveis pressóricos e a manutenção do tratamento contínuo como forma de controle da pressão arterial. Assim, o conhecimento foi avaliado como insatisfatório para o atendimento desses aspectos. A falta de orientação pelos profissionais da saúde sobre os níveis tensionais pode ter corroborado com um menor conhecimento sobre estes parâmetros. A literatura mostra que apesar dos médicos incentivarem a monitoração domiciliar, somente em $15 \%$ das consultas os portadores de HAS são orientados sobre os valores tensionais considerados normais 38 . Os achados deste estudo têm relevância no âmbito da saúde pública, uma vez que apontam para a necessidade de atuação interdisciplinar em programas e ações para o controle da HAS. Dessa forma, é necessária a reorganização do tempo dedicado pela equipe de saúde à orientação dos portadores quanto a aspectos fundamentais para o manejo da HAS, bem como a conscientização dos profissionais no que concerne à qualidade e clareza da orientação dada, principalmente, para indivíduos que dependem dos serviços públicos de saúde para ter acesso a consultas médicas e a medicamentos e que possuem, na sua maioria, nível de escolaridade inferior ao do profissional de saúde. 


\section{Resumen}

Este estudio objetivó evaluar el conocimiento sobre la hipertensión arterial (HTA) y los factores asociados. Estudio transversal con usuários de fármacos antihipertensivos que obtuvieron sus medicamentos en una farmacia comunitaria de São Francisco de Paula, Rio Grande do Sul, Brasil. Se evaluó los conocimientos acerca de los niveles de presión arterial sistólica y diastólica y de la condición crónica de la HTA. Se utilizó la regresión multinomial para evaluar el conocimiento (insuficiente/parcialmente suficiente/ suficiente), considerado conocimiento suficiente cuando el usuario sabía dos o más de los aspectos evaluados. De los 635 usuários, 27,7\% tenían un conocimiento suficiente y sólo $47,2 \%$ conocían la cronicidad de la HTA. El sexo femenino, la educación superior, no vivir solo, el comportamiento saludable y el tiempo de diagnóstico se asociaron con el conocimiento suficiente. Considerando la importancia de la autonomía del usuário en el monitoreo de la presión arterial y la adesión al tratamiento en el control de la HTA, se ha encontrado una baja prevalencia de conocimiento suficiente.

Anti-Hipertensivos; Atención Primaria de Salud; Presión Arterial; Conocimiento, Actitudes y Práctica en Salud

\section{Colaboradores}

F. R. Motter revisou a literatura, elaborou o projeto e instrumento de coleta de dados, realizou o treinamento das entrevistadoras, supervisionou o trabalho de campo, trabalhou na análise dos dados e na redação do artigo. M. T. A. Olinto participou da elaboração do projeto e instrumento de coleta de dados, análise dos dados e redação do artigo final. V. M. V. Paniz coordenou todas as etapas do projeto, da concepção, delineamento, análise dos dados até a redação final do artigo. Todos os autores trabalharam na redação do artigo, revisaram criticamente e aprovaram a versão final.

\section{Agradecimentos}

O projeto teve apoio financeiro do $\mathrm{CNPq}$ (processo no 478810/2010-5).

\section{Referências}

1. Departamento de Atenção Básica, Secretaria de Atenção à Saúde, Ministério da Saúde. Hipertensão arterial sistêmica para o Sistema Único de Saúde. Brasília: Ministério da Saúde; 2006.

2. Chobanian AV, Bakris GL, Black HR, Cushman WC, Green LA, Izzo Jr. JL, et al. The Seventh Report of the Joint National Committee on Prevention, Detection, Evaluation, and Treatment of High Blood Pressure: the JNC 7 report. JAMA 2003; 289: 2560-72.

3. Lewington S, Clarke R, Qizilbash N, Peto R, Collins R. Age-specific relevance of usual blood pressure to vascular mortality: a meta-analysis of individual data for one million adults in 61 prospective studies. Lancet 2002; 360:1903-13.

4. Chrestani MA, Santos IS, Matijasevich AM. Hipertensão arterial sistêmica auto-referida: validação diagnóstica em estudo de base populacional. Cad Saúde Pública 2009; 25:2395-406.

5. Piccini RX, Victora CG. How well is hypertension managed in the community? A population-based survey in a Brazilian city. Cad Saúde Pública 1997; 13:595-600.
6. Longo GZ, Neves J, Luciano VM, Peres MA. Prevalência de níveis pressóricos elevados e fatores associados em adultos de Lages/SC. Arq Bras Cardiol 2009; 93:387-94.

7. Law MR, Morris JK, Wald NJ. Use of blood pressure lowering drugs in the prevention of cardiovascular disease: meta-analysis of 147 randomised trials in the context of expectations from prospective epidemiological studies. BMJ 2009; 338:b1665.

8. Sociedade Brasileira de Cardiologia; Sociedade Brasileira de Hipertensão; Sociedade Brasileira de Nefrologia. VI diretrizes brasileiras de hipertensão. Rev Bras Hipertens 2010; 13:1-66.

9. McManus RJ, Mant J, Roalfe A, Oakes RA, Bryan S, Pattison HM, et al. Targets and self monitoring in hypertension: randomised controlled trial and cost effectiveness analysis. BMJ 2005; 331:493.

10. Pierin AM, Marroni SN, Taveira LA, Bensenor IJ. Controle da hipertensão arterial e fatores associados na atenção primária em Unidades Básicas de Saúde localizadas na Região Oeste da Cidade de São Paulo. Ciênc Saúde Coletiva 2011; 16 Suppl 1:1389-400. 
11. Karaeren H, Yokusoglu M, Uzun S, Baysan O, Koz C, Kara B, et al. The effect of the content of the knowledge on adherence to medication in hypertensive patients. Anadolu Kardiyol Derg 2009; 9:183-8.

12. Morgado M, Rolo S, Macedo AF, Pereira L, CasteloBranco M. Predictors of uncontrolled hypertension and antihypertensive medication nonadherence. J Cardiovasc Dis Res 2010; 1:196-202.

13. Motter FR, Olinto MT, Paniz VM. Conhecimento sobre a farmacoterapia por portadores de hipertensão arterial sistêmica. Ciênc Saúde Coletiva 2013; 18:2263-74.

14. Neutzling MB, Rombaldi AJ, Azevedo MR, Hallal PC. Fatores associados ao consumo de frutas, legumes e verduras em adultos de uma cidade no Sul do Brasil. Cad Saúde Pública 2009; 25:2365-74.

15. World Health Organization. Diet, nutrition and the prevention of chronic diseases. Geneva: World Health Organization; 2003.

16. Brant R. Assessing proportionality in the proportional odds model for ordinal logistic regression. Biometrics 1990; 46:1171-8.

17. Williams R. Generalized ordered logit/partial proportional odds models for ordinal dependent variables. Stata J 2006; 6:58-82.

18. Victora CG, Huttly SR, Fuchs SC, Olinto MT. The role of conceptual frameworks in epidemiological analysis: a hierarchical approach. Int J Epidemiol 1997; 26:224-7.

19. Jesus ES, Augusto MAO, Gusmão J, Ortega K, Pierin AMG. Perfil de um grupo de Hipertensos: aspectos biossociais, conhecimentos e adesão ao tratamento. Acta Paul Enferm 2008; 21:59-65.

20. Gazmararian JA, Williams MV, Peel J, Baker DW. Health literacy and knowledge of chronic disease. Patient Educ Couns 2003; 51:267-75.

21. Oliveria SA, Chen RS, McCarthy BD, Davis CC, Hill $\mathrm{MN}$. Hypertension knowledge, awareness, and attitudes in a hypertensive population. J Gen Intern Med 2005; 20:219-25.

22. Sanne S, Muntner P, Kawasaki L, Hyre A, DeSalvo KB. Hypertension knowledge among patients from an urban clinic. Ethn Dis 2008; 18:42-7.

23. Viera AJ, Cohen LW, Mitchell CM, Sloane PD. High blood pressure knowledge among primary care patients with known hypertension: a North Carolina Family Medicine Research Network (NC-FMRN) study. J Am Board Fam Med 2008; 21:300-8.

24. Mion Jr. D, Pierin AM, Bensenor IM, Marin JC, Costa KR, Henrique LF, et al. Hipertensão arterial na cidade de São Paulo: prevalência referida por contato telefônico. Arq Bras Cardiol 2010; 95:99-106.

25. Alexander M, Gordon NP, Davis CC, Chen RS. Patient knowledge and awareness of hypertension is suboptimal: results from a large health maintenance organization. J Clin Hypertens (Greenwich) 2003; 5:254-60.

26. Moser M, Franklin SS. Hypertension management: results of a new national survey for the hypertension education foundation: Harris interactive. J Clin Hypertens (Greenwich) 2007; 9:316-23.

27. Sanchez CG, Pierin AM, Mion Jr. D. Comparação dos perfis dos pacientes hipertensos atendidos em Pronto-socorro e em tratamento ambulatorial. Rev Esc Enferm USP 2004; 38:90-8.
28. Subramanian U, Hofer TP, Klamerus ML, Zikmund-Fisher BJ, Heisler M, Kerr EA. Knowledge of blood pressure targets among patients with diabetes. Prim Care Diabetes 2007; 1:195-8.

29. Pierin AMG, Mion Jr. D, Fukushina JT, Pinto AR, Kaminaga MM. O perfil de um grupo de pessoas hipertensas de acordo com o conhecimento e a gravidade da doença. Rev Esc Enferm USP 2001; 35:11-8.

30. Busari OA, Olanrewaju TO, Desalu OO, Opadijo OG, Jimoh AK, Agboola SM, et al. Impact of patients' knowledge, attitude and practices on hypertension on compliance with antihypertensive drugs in a resource-poor setting. Turk Silahlı Kuvvetleri Koruyucu Hekim Bul 2010; 9:87-92.

31. Cheng S, Lichtman JH, Amatruda JM, Smith GL, Mattera JA, Roumanis SA, et al. Knowledge of blood pressure levels and targets in patients with coronary artery disease in the USA. J Hum Hypertens 2005; 19:769-74.

32. Samal D, Greisenegger S, Auff E, Lang W, Lalouschek W. The relation between knowledge about hypertension and education in hospitalized patients with stroke in Vienna. Stroke 2007; 38:1304-8.

33. Sturmer G, Dias-da-Costa JS, Olinto MT, Menezes AM, Gigante DP, Macedo S. O manejo não medicamentoso da hipertensão arterial sistêmica no Sul do Brasil. Cad Saúde Pública 2006; 22:1727-37.

34. Prugger C, Keil U, Wellmann J, Bacquer D, Backer G, Ambrosio GB, et al. Blood pressure control and knowledge of target blood pressure in coronary patients across Europe: results from the EUROASPIRE III survey. J Hypertens 2011; 29:1641-8.

35. Olinto MT, Willett WC, Gigante DP, Victora CG. Sociodemographic and lifestyle characteristics in relation to dietary patterns among young Brazilian adults. Public Health Nutr 2011; 14:150-9.

36. Familoni BO, Ogun SA, Aina AO. Knowledge and awareness of hypertension among patients with systemic hypertension. J Natl Med Assoc 2004; 96:620-4.

37. Camey SA, Torman VBL, Hirakata VN, Cortes RX, Vigo A. Bias of using odds ratio estimates in multinomial logistic regressions to estimate relative risk or prevalence ratio and alternatives. Cad Saúde Pública 2014; 30:21-9.

38. Bell RA, Kravitz RL. Physician counseling for hypertension: what do doctors really do? Patient Educ Couns 2008; 72:115-21.

Recebido em 17/Abr/2014

Versão final reapresentada em 05/Ago/2014

Aprovado em 29/Set/2014 